\title{
Syndrome du canal carpien : des causes rares et des formes associées derrière une affection commune et stéréotypée
}

\author{
Emmanuel Fournier \\ Département de Physiologie, Faculté de Médecine Pitié-Salpêtrière, Sorbonne Université \\ 91 Bd de l'Hôpital, 75013 Paris \\ emmanuel.fournier@sorbonne-universite.fr
}

\section{Résumé}

Le syndrome du canal carpien (SCC) est une affection trop fréquente pour ne pas interpeller quotidiennement le praticien, ne serait-ce que par son intrication à d'autres pathologies, causales ou d'association fortuite. La symptomatologie typique, faite de paresthésies et de douleurs de la main le matin au réveil, est liée à une atteinte du nerf médian dans l'espace confiné du canal carpien, le plus souvent par inflammation locale et ténosynovite des fléchisseurs des doigts (activité répétitive des mains). Le SCC peut cependant être secondaire à des situations (grossesse) ou des affections (œdème, hypothyroïdie...) qui en accusent la physiopathologie ordinaire ou qui provoquent des dépôts dans le canal (amyloses, mucopolysaccharidoses...). Par ailleurs le SCC est favorisé par toutes les neuropathies qui causent une fragilité nerveuse (diabète notamment). Il est parfois le premier signe de ces diverses affections, dont il peut permettre un diagnostic précoce. L'examen électroneuromyographique (ENMG) est un examen clé pour confirmer le diagnostic (ralentissement de la conduction motrice et sensitive du nerf médian dans le canal carpien, par démyélinisation locale), chercher une neuropathie prédisposante et des signes de gravité (réduction d'amplitude des signaux électrophysiologiques) qui témoignent d'une perte axonale. Dans les formes avec ralentissement de conduction isolé sans signe de gravité, un traitement médical par attelle ou infiltration peut être tenté. S’il n'apporte pas la guérison ou s'il existe des signes de gravité ou des douleurs insupportables, une intervention chirurgicale de décompression est indiquée. Qu'elle se fasse par voie traditionnelle ou endoscopique, elle apporte un soulagement rapide, voire immédiat.

Mots clés : syndrome du canal carpien, électroneuromyographie, diabète, amylose, douleur

\section{Carpal tunnel syndrome : rare causes and associated forms behind a common and stereotyped affection}

\section{Summary}

Carpal tunnel syndrome (CTS) is too common a condition not to daily interact with the practitioner, if only because of its entanglement to other pathologies, causal or chance association. The typical symptomatology, with hand paresthesia and morning pain upon waking, is related to a median nerve injury in the confined space of the carpal tunnel, more often by local inflammation and tenosynovitis of the finger flexors (repet- 
itive activity of the hands). SCC may be secondary to situations (pregnancy) or conditions (edema, hypothyroidism ...) which exaggerate the ordinary pathophysiology or cause deposits in the channel (amyloidosis, mucopolysaccharidoses ...). Otherwise, SCC is favored by all neuropathies that cause nerve fragility (especially diabetes). It is sometimes the first sign of these various affections of which it can allow early diagnosis. Electroneuromyographic examination (ENMG) is a key examination to confirm the diagnosis (slowing of sensitive and motor conduction of the median nerve through the carpal tunnel, due to local demyelination), to look for a predisposing neuropathy and for signs of seriousness (amplitude reduction of electrophysiological signals) that indicate axonal loss. In SCC forms with only slowed conduction without sign of seriousness, a splint or infiltration treatment may be attempted. If this medical treatment does not bring healing, or if there are signs of seriousness or unbearable pains, a decompression surgery is indicated. Whether it is performed traditionally or endoscopically, it provides fast relief, even immediate.

Keywords : carpal tunnel syndrome, electroneuromyography, diabetes, amyloidosis, pain treatment

\section{Introduction}

Bloquer le canal carpien, à la porte de la main, c'est bloquer une voie névralgique du toucher et de la préhension digitale. Par sa fréquence, avec une incidence annuelle d'environ 3/1000 en France (soit 200000 nouveaux cas par an), le syndrome du canal carpien (SCC) constitue la première cause nerveuse de douleurs et une source majeure d'irritations et de gênes quotidiennes dans la population [1,2]. D'un autre côté, le caractère bénin et assez aisément curable du SCC en fait un modèle d'exercice et de réussite de médecine curative, généralement à l'origine de satisfactions, par le soulagement apporté aux patients, et par la mobilisation coordonnée d'un réseau restreint de praticiens (médecin de premier recours, électrophysiologiste, chirurgien) [3]. Ces caractéristiques, de fréquence et de curabilité, font que le SCC n'échappe pas à la médecine interne et même l'intéresse, en venant émailler la prise en charge d'affections plus complexes et moins bénignes, ou bien égarer les diagnostics par des formes atypiques ou intriquées, ou encore dissimuler des causes rares sous une trop grande banalité.

\section{Forme clinique typique}

\section{Une sémiologie subjective stéréotypée}

L'expression clinique du SCC est remarquablement stéréotypée, faite de paresthésies intermittentes de la main, à type de fourmillements, d'engourdissements, de picotements, de décharges électriques..., mais aussi de douleurs et parfois de troubles vasomoteurs de la main (sensations de 
gonflement des doigts, changement de couleur de la main). Paresthésies et douleurs siègent dans le territoire anatomique du nerf médian, prédominent généralement sur la face palmaire des trois premiers doigts, mais sont parfois décrites comme prenant la main entière. Les douleurs irradient fréquemment à l'avant-bras ou au coude, plus rarement jusqu'à l'épaule. La symptomatologie est bilatérale dans un tiers des cas environ.

L'installation des signes subjectifs est le plus souvent progressive. Une caractéristique typique des paresthésies du SCC est d'être initialement matinales, au réveil, puis nocturnes, réveillant le malade et l'obligeant à se lever (acroparesthésies nocturnes). Le soulagement est obtenu en changeant de position, en secouant la main ou en effectuant des mouvements de flexion et d'extension répétée des doigts.

Dans la journée, paresthésies et douleurs peuvent être déclenchées ou réactivées par certains mouvements ou par le maintien de positions (poignet en hyperextension ou en hyperflexion, ou en appui de la face antérieure du carpe sur un plan dur), lors d'activités manuelles professionnelles ou de loisir (usage d'outils vibrants, utilisation répétitive de la pince pouce-index, travail à la souris d'ordinateur avec poignet en appui et en hyperextension, jardinage, bricolage...). L'évolution se fait vers des troubles sensitifs permanents, avec insomnie, maladresse puis faiblesse de la main.

\section{Physiopathologie}

La symptomatologie résulte de la compression ou de l'irritation du nerf médian dans le canal carpien $[3,4]$. Le passage de ce canal constitue un point à risque pour le nerf médian pour plusieurs raisons (Figure 1) : 1) étroitesse du canal (limité par les os du carpe en arrière et par le ligament annulaire antérieur du carpe en avant (avec une section transversale minimale située 2 à $3 \mathrm{~cm}$ audessous du pli du poignet) ; 2) cohabitation du nerf médian avec les tendons fléchisseurs du poignet et des doigts dans cet espace inextensible ; 3) grande mobilité du poignet (dont les mouvements étirent et compriment le nerf dans le canal étroit où il coulisse). Ces différents facteurs contribuent à induire une élévation de la pression intracanalaire, entretenant un œè̀me par stase veineuse et une ischémie qui perturbent le fonctionnement des fibres du nerf médian. Il existe un certain effet différentiel des facteurs physiopathologiques : l'œdème joue en premier sur les fibres nerveuses sensitives de gros calibre, sensibles à la compression (paresthésies), tandis que l'ischémie sollicite d'abord les fibres fines nociceptives (douleurs). Le rôle de la pression intracanalaire, variable en fonction de la position du poignet, explique que les symptômes soient déclenchés dans les circonstances susceptibles de renforcer l'œdème ou l'ischémie (positions prolongées, activités répétitives des mains, ténosynovites des fléchisseurs des doigts, grossesse...), tandis que les manœuvres ayant pour effet de réduire la stase veineuse et de drainer l'œdème apportent un soulagement. 


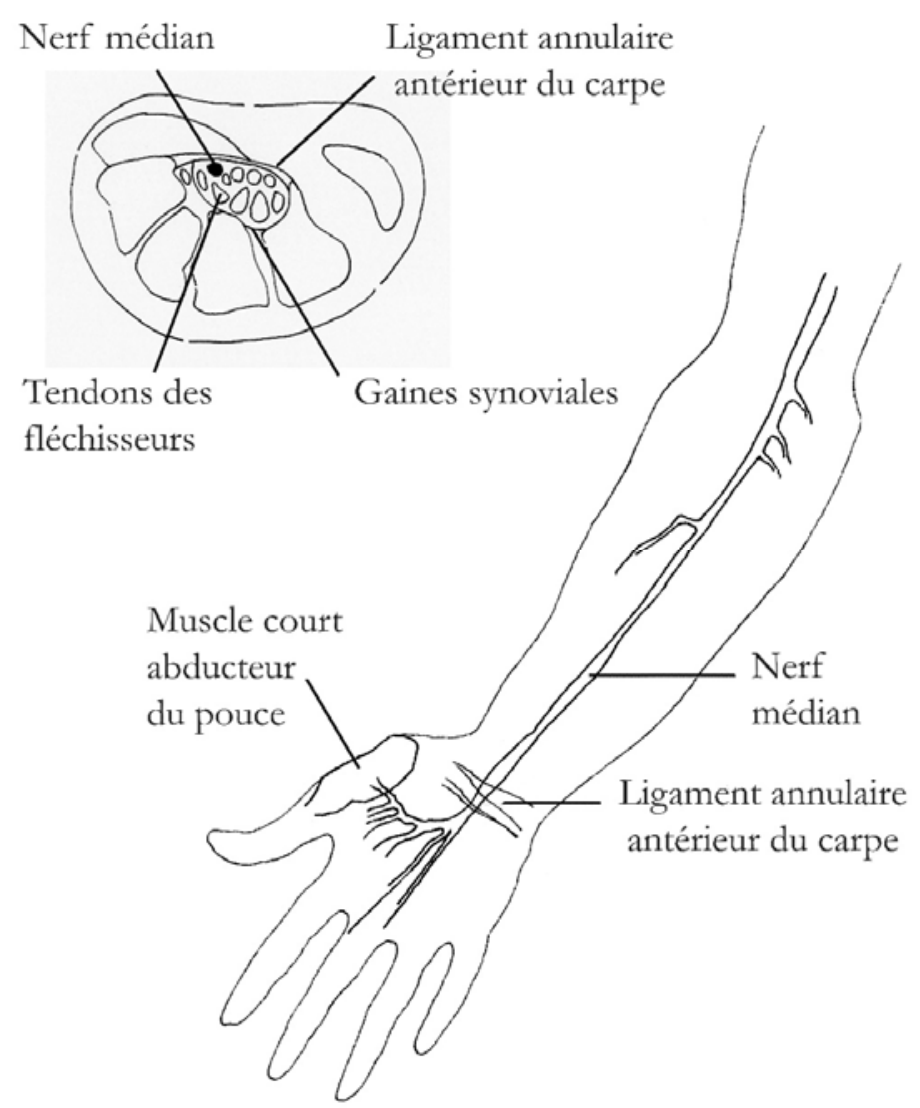

Figure 1. Schéma du trajet du nerf médian à l'avant-bras et au poignet, sous le ligament annulaire antérieur du carpe, avec coupe transversale du poignet montrant ses rapports dans le canal carpien.

L'atteinte du nerf peut être favorisée par des particularités anatomiques du canal (surface de section réduite avec une possible prédisposition génétique, épaississement du ligament annulaire, arthrose du poignet...) ou des particularités génétiques du tissu conjonctif [5,6,7]. Ces prédispositions contribuent à la compression progressive du nerf médian lorsqu'au fil des ans et des microtraumatismes (par mouvements répétitifs), s'installe une ténosynovite qui augmente le volume des tendons fléchisseurs des doigts. On explique ainsi que le SCC prédomine sur la main dominante dans $80 \%$ des cas, qu'il atteigne deux fois plus de femmes que d'hommes, ou qu'il survienne avec une fréquence maximale dans la cinquantaine et chez les sujets à poignet mince au regard de la longueur de la main. Le surpoids et l'obésité (avec IMC $>30 \mathrm{~kg} / \mathrm{m}^{2}$ ) sont considérés comme des facteurs favorisants [8]. 


\section{Examen clinique}

L'examen clinique peut être normal ou révéler un trouble de la sensibilité à type de dysesthésies (sensation d'engourdissement ou de décharge électrique au toucher), intéressant l'ensemble du territoire sensitif du nerf médian, c'est-à-dire les trois premiers doigts et la moitié latérale du $4^{e}$ doigt, mais se limitant parfois aux $2^{\mathrm{e}}$ et $3^{\mathrm{e}}$ doigts.

Les paresthésies peuvent être reproduites par la percussion de la face antérieure du carpe sur le trajet du nerf médian (signe de Tinel), ou bien par la flexion ou l'hyperextension passive forcée du poignet (signe de Phalen). Ces manœuvres provoquent anormalement facilement une sensation de décharge électrique irradiant dans le territoire médian à la main.

L'examen recherche en outre des signes de gravité qui témoignent d'une perte axonale et qui influencent la prise en charge thérapeutique :

- perte de sensibilité dans le territoire nerveux du médian,

- faiblesse des muscles thénariens dépendant du nerf médian, c'est-à-dire principalement du court abducteur du pouce (déficit de l'antépulsion contrariée du pouce), mais aussi de l'opposant du pouce (déficit de l'opposition pouce-auriculaire), avec, dans les formes avancées, amyotrophie le long du premier métatarsien (à distinguer d'une pseudo-paralysie et d'une pseudo-amyotrophie par rhizarthrose du pouce).

\section{Diagnostic différentiel}

Même si le canal carpien représente le site le plus fréquent d'atteinte du nerf médian, celui-ci peut être lésé en des points plus proximaux de son trajet, notamment à la partie haute de l'avant-bras (syndrome du rond pronateur), ou même plus haut encore au bras, ou à l'aisselle. Le diagnostic repose sur l'analyse topographique des branches nerveuses touchées.

Dans le syndrome du rond pronateur, le nerf médian est atteint dans sa traversée du muscle rond pronateur [9]. L'atteinte à ce niveau est favorisée par des activités intenses de l'avant-bras et de la main, avec pronation vigoureuse et prolongée (pratique de la planche à voile ou de la moto) ou par un syndrome des loges de l'avant-bras. Un traumatisme, une fracture du coude ou une atteinte ischémique par vascularite peuvent aussi être en cause.

Dans ces cas, les troubles sensitifs (et éventuellement moteurs) en territoire médian à la main, sont en tous points semblables à ceux du SCC, mais s'associent à des signes qui attirent l'attention vers une atteinte au coude : douleurs et sensibilité à la pression de la partie haute de l'avant-bras ou du coude ; faiblesse des muscles du territoire médian à l'avant-bras (fléchisseur radial du carpe, fléchisseurs superficiel et profond des doigts) [10]. Le rond pronateur lui-même (dont le nerf propre se détache du nerf médian en amont du site lésionnel) est le plus souvent épargné dans le syndrome 
du rond pronateur. Il est au contraire déficitaire dans les atteintes au coude, au bras et à l'aisselle (luxation antérieure de l'épaule ; compression externe par béquilles axillaires...).

Certaines formes dissociées (sensitives ou motrices) de SCC peuvent prêter à confusion avec des atteintes radiculaires ou plexiques : atteinte de la racine C6 (ou du cordon plexique supérieur) avec paresthésies du pouce seul (et faiblesse du brachioradial), atteinte de la racine C7 (ou du cordon plexique moyen) avec paresthésies de l'index et du majeur (et faiblesse du triceps), atteinte de la racine C8 (ou du cordon plexique inférieur, syndrome du défilé cervico-brachial) avec amyotrophie en territoire médian à la main et troubles sensitifs à la face médiale de l'avant-bras et des deux derniers doigts.

\section{Diagnostic électro(neuro)myographique}

L'électro(neuro)myographie (EMG ou ENMG) permet à la fois de confirmer l'atteinte du nerf médian dans le canal carpien, d'éliminer les diagnostics différentiels et de mettre en évidence d'éventuels signes de gravité ou atteintes neuromusculaires associées. L'usage de l'électrophysiologie à ces fins est fiable et ancien, sans véritable technique concurrente depuis ses débuts en 1956 [11]. L'échographie et les autres techniques d'imagerie montrent des images intéressantes (épaississement ou aplatissement du nerf médian, épaississement des ligaments et des tendons fléchisseurs), mais n’aident véritablement ni le diagnostic ni la décision thérapeutique.

D’innombrables variantes de techniques électrophysiologiques ont été décrites, avec des résultats non très différents [12]. Toutes ont pour principe commun de mettre en évidence un ralentissement de la conduction sensitive et de la conduction motrice du nerf médian dans le canal carpien [10]. Un tel ralentissement tient à la fois à une démyélinisation focale des fibres nerveuses et à une réduction de leur calibre, toutes deux induites par la compression. La stratégie générale d'examen repose sur une étude comparative des nerfs médians et ulnaires droits et gauches par électrodes de surface, éventuellement complétée par un examen EMG à l'aiguille d'un ou deux muscles. L'ensemble est rapide et peut être rendu indolore, si l'on en prend soin.

\section{Ralentissement de la conduction nerveuse sensitive et motrice}

Le principe de l'étude de la conduction sensitive est de stimuler une branche sensitive du nerf médian (telle que le deuxième nerf interosseux qui innerve les faces opposées de l'index et du majeur) par un courant électrique de faible intensité, et d'enregistrer, par des électrodes de surface collées à la surface du poignet, le potentiel sensitif conduit de l'autre côté du canal carpien (Figure 2). Le premier signe de SCC est une diminution de la vitesse de conduction sensitive (VCS) transcanalaire du nerf médian : elle témoigne d'un ralentissement de la conduction des plus grosses fibres sensitives du nerf, qui peut rendre compte des dysesthésies. Le ralentissement s'apprécie par rapport aux valeurs 
de VCS de référence dans la population (normalement de l'ordre de $50-55 \mathrm{~m} / \mathrm{s},>45 \mathrm{~m} / \mathrm{s}$ ), et de façon plus sensible par comparaison à la VCS du nerf ulnaire voisin (normale dans le SCC), mesurée dans des conditions semblables.

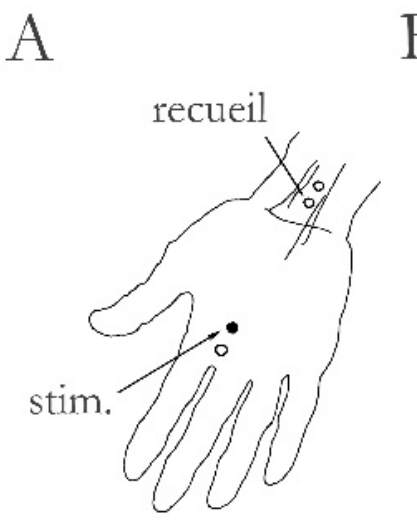

\section{$\mathrm{B}$}

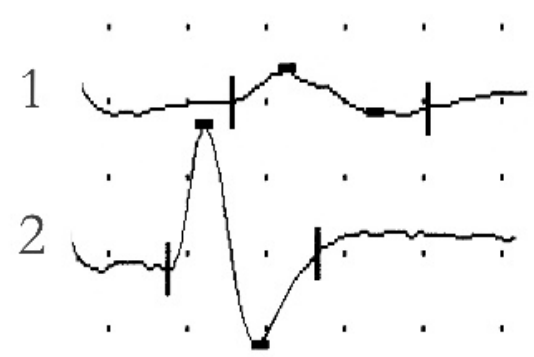

Figure 2. Étude de la conduction sensitive du nerf médian. $\boldsymbol{A}$ : Schéma de positionnement des électrodes de stimulation à la paume, et de recueil au poignet. $\boldsymbol{B}$ : Potentiels sensitifs des nerfs médians droit et gauche. 1: à droite, SCC sévère avec diminution de VCS $(34 \mathrm{~m} / \mathrm{s})$ et signe de gravité sous forme d'une réduction d'amplitude du potentiel sensitif $(6,1 \mu \mathrm{V})$. 2: à gauche, SCC minime avec simple diminution de VCS $(42 \mathrm{~m} / \mathrm{s})$ sans réduction d'amplitude du potentiel sensitif $(29,4 \mu \mathrm{V})$. Échelle : $10 \mu \mathrm{V}-1 \mathrm{~ms} / \mathrm{div}$.

L'étude de la conduction nerveuse motrice consiste à délivrer une stimulation électrique au nerf médian au poignet (en amont du canal carpien) et à enregistrer par des électrodes de surface la réponse motrice induite en aval, sur le muscle court abducteur du pouce (Figure 3). Le SCC se marque par un allongement de la latence distale d'apparition de la réponse, (normalement de l'ordre de $3 \mathrm{~ms},<3,7$ ms) : cet allongement reflète le ralentissement de la conduction des fibres nerveuses motrices à travers le canal carpien. Si le nerf peut être stimulé à la paume, l'étude montre l'existence d'une diminution de la vitesse de conduction motrice (VCM) poignet-paume (transcanalaire).

En général, le ralentissement de la conduction sensitive est plus précoce et plus sensible que l'allongement de latence distale motrice [10,13]. Les fibres sensitives de plus gros calibre, légèrement plus grosses que les plus grosses des fibres motrices, sont atteintes les premières par la compression. Cependant, il existe des formes dissociées, avec atteinte à divers degrés des branches du médian : atteinte sélective de la branche motrice thénarienne avec amyotrophie et allongement de la latence distale du nerf médian, sans anomalie de conduction sensitive ; atteinte sélective de certains nerfs sensitifs interdigitaux, avec paresthésies dans un territoire médian réduit et altérations des potentiels sensitifs doigt-poignet correspondants. 
A

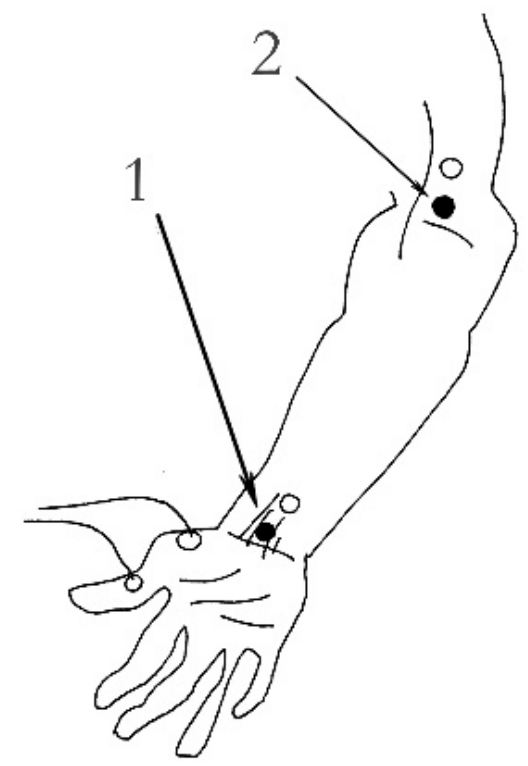

B

$\cdot, \cdot$

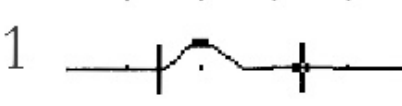

2

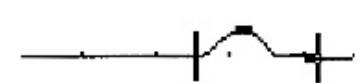

C

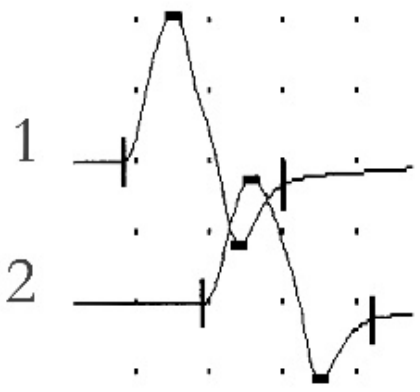

Figure 3. Étude de la conduction motrice du nerf médian par stimulation au poignet (1) et au coude (2), recueil sur le muscle court abducteur du pouce. $\boldsymbol{A}$ : à droite, SCC sévère avec allongement de latence distale marqué (7,1 ms) et signe de gravité sous forme d'une réduction d'amplitude de la réponse motrice $(2,0 \mathrm{mV})$. $\boldsymbol{B}:$ à gauche, SCC minime avec allongement de latence distale (4,1 ms) sans réduction d'amplitude $(16,5 \mathrm{mV})$. Échelle : $5 \mathrm{mV}-5 \mathrm{~ms} /$ div.

\section{Signes de gravité}

Au stade de ralentissement simple, le SCC est souvent réversible, la gaine myélinique pouvant assez facilement se refaire dès lors que la compression est levée. Il en va autrement si, outre le ralentissement de conduction, l'étude de la conduction nerveuse motrice et sensitive met en évidence une réduction de l'amplitude des réponses enregistrées (normalement $>15 \mu \mathrm{V}$ pour les potentiels sensitifs, $>6 \mathrm{mV}$ pour les réponses motrices). De telles réductions d'amplitude témoignent le plus souvent d'une dégénérescence axonale qui constitue un élément de gravité dans la mesure où elle entraîne une perte de fonction et où elle rend la récupération longue, aléatoire et souvent incomplète. Elles font partie des critères d'indication d'une intervention chirurgicale. Dans la progression ordinaire d'un SCC, l'amplitude des potentiels sensitifs baisse après une période de simple ralentissement de conduction, et avant que ne baisse l'amplitude des réponses motrices.

L'existence d'une perte axonale motrice peut également être appréciée par un examen EMG de détection du muscle court abducteur de pouce. L'insertion d'une aiguille fine dans ce muscle met 
alors en évidence des signes de dénervation musculaire au repos (fibrillation) et, lors de la contraction volontaire du muscle, un manque de potentiels d'unité motrice (tracé pauvre) avec signes de réinnervation collatérale (augmentation d'amplitude des potentiels d'unité motrice restants). Cependant, cet enregistrement à l'aiguille est rarement nécessaire, car les études de la conduction nerveuse par électrodes de surface peuvent généralement révéler (de façon non invasive) une perte axonale sous forme d'une réduction de l'amplitude des réponses.

\section{Signes négatifs - Diagnostic différentiel des atteintes hautes du médian}

Outre les signes positifs de SCC et les signes de gravité, l'un des objectifs de l'examen ENMG est d'éliminer les diagnostics différentiels de paresthésies des doigts en territoire médian [10]. L'étude de la conduction nerveuse motrice du nerf médian à l'avant-bras vérifie l'absence d'anomalie audessus du poignet (Figure 3). Si l'examen clinique laisse un doute sur un syndrome du rond pronateur ou sur une atteinte plus haute du nerf médian, l'examen EMG à l'aiguille recherche des signes d'atteinte neuropathique dans un muscle du territoire médian à l'avant-bras (fléchisseur radial du carpe) et éventuellement dans le rond pronateur.

Si les paresthésies du pouce ou de l'index et du majeur font suspecter une atteinte radiculaire, l'examen EMG peut être amené à explorer les muscles brachioradial et triceps, à la recherche d'une atteinte des racines $\mathrm{C} 6$ et $\mathrm{C} 7$, respectivement.

Par ailleurs, l'examen comprend systématiquement une étude de la conduction nerveuse motrice et sensitive du nerf ulnaire, voire un examen EMG à l'aiguille d'un muscle en territoire C8 à la main ( $1^{\text {er }}$ interosseux), à la recherche d'une atteinte de cette racine ou du cordon inférieur du plexus brachial, qui pourrait être la cause d'une amyotrophie thénarienne.

\section{Formes cliniques et électrophysiologiques}

\section{Formes asymptomatiques}

Du fait de son incidence élevée, le SCC est une découverte fréquente d'examens ENMG pratiqués pour d'autres indications. Parfois l'entretien avec le patient retrouve a posteriori des symptômes de SCC négligés. L'association avec l'affection motivant l'exploration ENMG peut être fortuite ou s'avérer révélatrice d'une affection causale ou d'une neuropathie (voir plus loin).

Outre ces formes minimes ou débutantes de SCC, il existe des formes chroniques insidieuses (souvent du sujet âgé), d’installation très lentement progressive, sans douleurs ni paresthésies intenses. C’est un examen clinique ou électrophysiologique qui découvre incidemment une amyotrophie du court abducteur du pouce, parfois sévère, avec anomalies électrophysiologiques sensitives et motrices plus marquées que ne le laissaient prévoir les signes subjectifs cliniques. 


\section{Formes hyperalgiques}

Chez certains patients, la symptomatologie est faite avant tout de douleurs intenses, sans les riches paresthésies typiques du SCC. L'examen électrophysiologique est décevant, normal ou ne montrant que des anomalies relativement minimes. On peut supposer que, dans ces formes hyperalgiques, le mécanisme d'ischémie, auquel sont particulièrement sensibles les fibres nociceptives fines, l'emporte sur l'œdème et ses effets compressifs sur les grosses fibres sensitives. Comme les signaux électrophysiologiques, notamment les potentiels sensitifs, n'enregistrent que les fibres de gros calibre, les atteintes des fibres de la douleur, de petit calibre, leur échappent.

Les formes ischémiques hyperalgiques de SCC sont très sensibles à une libération chirurgicale : la levée de la compression ischémique entraîne une disparition instantanée de la symptomatologie douloureuse, évidente dès le réveil de l'intervention.

\section{Formes extensives ou amplifiées}

Le canal carpien est parfois, sinon à l'origine, du moins partie prenante de tableaux de douleurs mal systématisées, parfois impossibles à localiser (patients disant «avoir mal partout»), en particulier dans les tableaux dits de «fibromyalgie» [14]. Quelque idée qu'on se fasse de la physiopathologie de ces tableaux, l'examen clinique et électrophysiologique peut se donner comme objectif de mettre en évidence et d'individualiser des points d'appel douloureux, dont l'association crée ou contribue à créer la sensation de douleur diffuse. La mise en évidence d'un SCC, dont les douleurs se fondent dans la douleur diffuse, donne au clinicien une possibilité de localiser celle-ci, de la diviser, et d'entrer avec le patient dans une dynamique thérapeutique à partir de ce point d'action.

\section{Formes étiologiques}

Le SCC est dû, dans la grande majorité des cas, à une élévation chronique de la pression intracanalaire, par rétrécissement du canal, inflammation locale et ténosynovite des fléchisseurs des doigts. Ce caractère «idiopathique » très habituel ne doit pas faire oublier que le SCC peut être lié à un traumatisme ou secondaire à une cause locale ou plus générale.

Les atteintes traumatiques aiguës du nerf médian dans le canal carpien sont habituellement les suivantes : étirement par chute avec hyperextension des poignets, écrasement, blessure par plaie pénétrante, fracture des os du carpe ou fracture de Pouteau-Colles. On en rapproche les atteintes compressives par immobilisation plâtrée ou dans le cadre d'un syndrome des loges.

Parmi les causes locales, on compte les déformations osseuses post-traumatiques (cals vicieux...); les tumeurs intracanalaires (kyste synovial des tendons fléchisseurs des doigts, neurinome, lipome); les anomalies musculaires (avec présence intracanalaire des corps musculaires des lombricaux ou 
des fléchisseurs ou des doigts); les hémorragies et hématomes intracanalaires (d'origine traumatique ou liées à un trouble de l'hémostase), les thromboses de l'artère du nerf médian. Ces causes locales représentent les indications de l'imagerie (radiographie, scanographie, IRM ou échographie) dans l'exploration d'un SCC. En pratique cependant, elles sont ou évidentes ou trop rares pour faire l'objet d'une recherche systématique.

En revanche, la présence d'une cause plus générale, dont le SCC est parfois révélateur, doit être systématiquement évoquée. Ce sont en premier lieu toutes les situations susceptibles de provoquer une infiltration ou une inflammation des structures du canal carpien.

Dans les causes hormonales, on retient d'une part la grossesse (qui peut être à l'origine de formes très aiguës et invalidantes) $[15,16]$, et d'autre un certain nombre d'affections endocriniennes : l'hypothyroïdie (en ce cas, l'hormonothérapie substitutive suffit en général à traiter le syndrome du canal carpien), l'acromégalie et l'hyperparathyroïdie $[17,18]$.

D’autre causes de SCC sont à rechercher du côté des maladies conduisant à des dépôts intracanalaires, rétrécissant sa section : l'amylose (héréditaire ou acquise, avec myélome multiple) est ainsi une pourvoyeuse de SCC, par ses dépôts amyloïdes [19,20,21] ; de même les mucopolysaccharidoses, par dépôts de mucopolysaccharides, à l'origine de formes infantiles de SCC, détectables dès l'âge de 3 ans, parfois très sévères; ou encore la sarcoïdose [22].

Par ailleurs, la physiopathologie du SCC explique qu'il soit favorisé par les ténosynovites spécifiques de la polyarthrite rhumatoïde, du lupus érythémateux disséminé, de la sclérodermie et de la goutte [23].

Peuvent être aussi responsables de SCC les vascularites qui prennent pour cible la vascularisation fragile du nerf médian dans le canal carpien; ou l'hémodialyse avec ischémie en aval du site de dialyse $[24,25]$.

Cependant, l'incidence élevée du SCC dans la population fait qu'il survient fréquemment au cours de l'évolution d'autres affections, sans qu'il y ait de relation causale.

\section{Formes révélatrices de neuropathies}

Outre les causes à proprement parler de SCC, les neuropathies diffuses constituent des facteurs de prédisposition au SCC. Celui-ci s’inscrit alors dans leur tableau. Par le point de fragilité mécanique qu'il représente, le canal carpien fonctionne comme un lieu d'expression première et de détection précoce des fragilités nerveuses. Le SCC est ainsi plus fréquent dans toute polyradiculonévrite démyélinisante, qu'elle soit aiguë (syndrome de Guillain-Barré) ou chronique, et dans les polyneuropathies (y compris classées «axonales ») qui ne laissent pas la myéline indemne. Son incidence est ainsi particulièrement élevée dans le diabète (30\% chez les diabétiques ayant une polyneuropathie, 
14\% chez ceux qui n'en ont pas) [26] et dans les neuropathies héréditaires avec hypersensibilité à la pression, où les altérations de conduction du nerf médian au canal carpien ne manquent jamais à l'examen ENMG [27].

Les formes de SCC «associées » ou «favorisées par des neuropathies » justifient qu'en plus des nerfs médians, l'examen électrophysiologique explore systématiquement au moins les nerfs ulnaires, et, au moindre doute, les nerfs des membres inférieurs, à la recherche d'une neuropathie plus diffuse dont le SCC ne serait que la « partie émergée ».

\section{Prise en charge}

L'évolution naturelle du SCC se fait vers une disparition spontanée des symptômes dans un tiers des cas environ, ce qui incite à l'abstention thérapeutique ou à de simples manœuvres ergonomiques dans les cas débutants et peu sévères [28].

Les indications des traitements dépendent de la présence ou non de signes de gravité [29,30,31]. Ceux-ci sont avant tout les signes de perte axonale recueillis par l'examen clinique (déficit sensitif objectif, amyotrophie du muscle court abducteur du pouce) ou l'examen ENMG (réduction d'amplitude des réponses électrophysiologiques, éventuellement fibrillation musculaire).

Les traitements médicaux, par attelle ou infiltration de corticoïdes, sont indiqués en première intention en l'absence de signe de gravité, ou bien comme solution d'attente avant un traitement chirurgical, ou encore lorsqu'une résolution spontanée est prévisible (grossesse, sollicitation intensive inhabituelle de la main) [32]. Le SCC régresse après l'accouchement et à l'arrêt d'une activité manuelle causale temporaire.

Dans les cas de SCC secondaires, le traitement de la cause est essentiel. L'existence d'une telle cause ou d'une neuropathie prédisposante ne dispense pas de la discussion d'une intervention chirurgicale dans les cas sévères avec perte axonale.

\section{Mesures ergonomiques}

Dans tous les cas, il faut essayer d'apprécier le rôle des activités manuelles favorisant la douleur et les paresthésies. L'objectif est de mettre en place des mesures préventives visant à réduire les microtraumatismes répétés. Si les activités manuelles causales peuvent être suspendues, il arrive, dans un nombre non négligeable de cas, que la symptomatologie disparaisse [33,34].

Les atteintes professionnelles peuvent entrainer un aménagement du poste et des outils de travail (outils non vibrants, tapis de souris ergonomique avec repose-poignet...), l'instauration de périodes de repos, une diversification des activités manuelles (rotation de poste) ou un changement d'affectation. En France, le SCC figure au tableau des maladies professionnelles (sous le $n^{\circ} 57$ du régime 
général de la sécurité sociale) et peut donner lieu à des indemnisations. Il est le plus fréquent des «troubles musculo-squelettiques ».

\section{Orthèses (attelles)}

Les orthèses en coutil baleiné, ou thermo-moulées sur mesure, immobilisent le poignet en position neutre. Elles peuvent être portées seulement la nuit. Dans les formes légères ou modérées, elles permettent souvent de calmer les symptômes nocturnes. Leur efficacité est jugée après 3 mois $[32,33]$.

\section{Infiltrations}

L'injection d'un dérivé corticoïde dans le canal carpien apporte en quelques jours un soulagement, voire la disparition complète de tous les symptômes. L'efficacité peut durer plusieurs semaines, mois ou années, évitant l'intervention chirurgicale, mais elle n'est souvent que temporaire : la récidive à moyen ou long terme toucherait 75 à $90 \%$ des patients [35]. L'infiltration peut éventuellement être répétée au bout de trois mois, mais en cas d'échec (soulagement incomplet ou non persistant), la question d'une intervention chirurgicale de décompression se pose [36,37,38]. L'infiltration n'est pas sans risque. La technique doit être pratiquée de manière rigoureuse pour éviter une injection intraneurale qui causerait des lésions irréversibles du nerf médian. Elle peut être éventuellement guidée par échographie.

\section{Chirurgie}

Le traitement chirurgical est proposé soit d'emblée lorsque des signes de gravité cliniques ou électrophysiologiques sont présents, soit après échec du port d'une attelle nocturne (pendant au moins trois mois) ou de deux infiltrations (espacées de trois à six mois), soit dans les formes ischémiques hyperalgiques avec douleurs insupportables [39,40,41].

L'acte chirurgical principal consiste à sectionner le ligament annulaire antérieur du carpe, de façon à donner de l'espace au contenu du canal carpien, nerf médian et tendons fléchisseurs. Les complications sont rares (section d'un rameau nerveux, algodystrophie, infection) et les suites post-opératoires sont en règle générale simples. L'intervention est réalisée le plus souvent en chirurgie ambulatoire, sous anesthésie locorégionale et sous garrot pneumatique. Elle peut se faire soit par chirurgie conventionnelle, dite «à ciel ouvert » (une incision de deux ou trois centimètres en haut de la paume de la main) soit par voie endoscopique (une incision d'un centimètre au poignet avec éventuellement une deuxième incision dans la paume de la main).

Il n'a pas été démontré de différence d'efficacité clinique à moyen et long terme pour les techniques chirurgicales à ciel ouvert ou par voie endoscopique [42,43]. La seconde a des suites immédiates 
généralement plus simples dans les premières semaines, mais comporte un plus grand risque de lésion du nerf médian. La chirurgie à ciel ouvert permet de réaliser des gestes complémentaires sur les tendons des fléchisseurs (synovectomie) ou sur le nerf médian (neurolyse, épineurotomie).

Le poignet n'est pas immobilisé en post-opératoire. Un repos prolongé est cependant nécessaire avant la reprise d'un travail manuel exigeant. Une rééducation est utile (manipulation d'une balle en mousse). La durée d'arrêt de travail suite à une intervention chirurgicale du canal carpien est au minimum de 7 jours, davantage en cas de travail physique intense $[44,45]$.

Quelle que soit la technique, les résultats sont généralement bons. L'intervention soulage immédiatement la douleur, notamment dans les formes hyperalgiques. Il en résulte que des douleurs intenses peuvent constituer à elles seules une indication chirurgicale, même s'il n'y a pas d'autre signe de gravité et même si l'examen clinique et électrophysiologique est normal.

Les troubles sensitifs permanents disparaissent généralement en quelques semaines (le temps d'une remyélinisation). Ce n'est qu'en cas de dégénérescence axonale (avec atrophie et parésie initiales des muscles thénariens) que la récupération postopératoire peut être partielle et longue, voire absente. La qualité de la récupération dépend également de l'âge du patient, de l'ancienneté de la compression ainsi que de la présence de facteurs défavorables comme travail manuel intense, diabète ou autre neuropathie.

À distance de l'intervention, la majorité des sujets opérés se considèrent comme guéris. Seuls certains se plaignent de douleurs liées à la cicatrice, ou de quelques douleurs résiduelles dans le territoire médian à la main, ou encore d'une légère perte de force de préhension.

Alors que les récidives ne sont pas rares après traitement médical, elles sont exceptionnelles après chirurgie (section incomplète du ligament). La réapparition ou la persistance de la symptomatologie après chirurgie impose de refaire un examen clinique et ENMG (à 3 mois) et de discuter une reprise chirurgicale.

Le diagnostic et la prise en charge du SCC sont donc simples et satisfaisants dans la majorité des cas. Cependant, aussi «banal » soit-il, le syndrome ne manque pas de sel pour qui sait s'intéresser, derrière le commun, à la variété des vécus humains et au jeu rusé d'affections causales moins communes dont il est parfois la première manifestation. À part le diabète, lui-même fréquent, ces causes sont trop rares pour justifier de toutes les rechercher de façon systématique et approfondie. Elles n'en appellent pas moins à conserver une vigilance.

\section{Conflits d'intérêts : aucun.}




\section{Références bibliographiques}

1. Latinovic R, Gulliford MC, Hughes RA. Incidence of common compressive neuropathies in primary care. J Neurol Neurosurg Psychiatry 2006, $77:$ 263-5.

2. Burton CL, Chen Y, Chesterton LS, Van der Windt DA. Trends in the prevalence, incidence and surgical management of carpal tunnel syndrome between 1993 and 2013 : an observational analysis in UK primary care records. BMJ Open 2018, 8 : e020166.

3. Gelfman R, Melton LJ 3rd, Yawn BP, Wollan PC, Amadio PC, Stevens JC. Long-term trends in carpal tunnel syndrome. Neurology 2009, 72:33-41.

4. Urits I, Gress K, Charipova K, Orhurhu V, Kaye AD, Viswanath O. Recent advances in the understanding and management of carpal tunnel syndrome: a comprehensive review. Curr Pain Headache Rep 2019, $23: 70$.

5. Wiberg A, Ng M, Schmid AB, Smillie RW, Baskozos G, Holmes MV et al. A genome-wide association analysis identifies 16 novel susceptibility loci for carpal tunnel syndrome. Nat Commun 2019, $10: 1030$.

6. Hakim AJ, Cherkas L, El Zayat S, MacGregor AJ, Spector TD. The genetic contribution to carpal tunnel syndrome in women : a twin study. Arthritis Rheum 2002, $47: 275-9$.

7. Dada S, Burger MC, Massij F, de Wet H, Collins M. Carpal tunnel syndrome : The role of collagen gene variants. Gene 2016, $587:$ 53-8.

8. Nathan PA, Keniston RC, Myers LD, Meadows KD. Obesity as a risk factor for slowing of sensory conduction of the median nerve in industry. A cross-sectional and longitudinal study involving 429 workers. J Occup Med 1992, $34:$ 379-83.

9. Gessini L, Jandolo B, Pietrangeli A. The pronator teres syndrome. Clinical and electrophysiological features in six surgically verified cases. J Neurosurg Sci 1987, $31: 1-5$.

10. Fournier E. Syndrome du canal carpien. In : Fournier E, éd. Électromyographie, Vol. 4. Syndromes EMG d'atteinte des nerfs et des muscles, Paris : Lavoisier, 2013. p. 47-55.

11. Simpson JA. Electrical signs in the diagnosis of carpal tunnel and related syndromes. J Neurol Neurosurg Psychiatr 1956, $19: 275-280$.

12. American Association of Electrodiagnostic Medicine, American Academy of Neurology, and American Academy of Physical Medicine and Rehabilitation. Practice parameter for electrodiagnostic studies in carpal tunnel syndrome : summary statement. Muscle Nerve 2002, 25 : 918-22.

13. Demino C, Fowler JR. The sensitivity and specificity of nerve conduction studies for diagnosis of carpal tunnel syndrome : a systematic review. Hand (N Y)_2019 : 1558944719855442.

14. Ginanneschi F, Filippou G, Adinolfi A, Di Sabatino V, Frediani B, Rossi A. Spasmophilia and entrapment nerve syndrome comorbidities in fibromyalgic patients : a possible neuromuscular pain generator. Reumatismo 2018, 70 : 251-256.

15. Wright C, Smith B, Wright S, Weiner M, Wright K, Rubin D. Who develops carpal tunnel syndrome during pregnancy : An analysis of obesity, gestational weight gain, and parity. Obstet Med 2014, 7 : 90 4. 
16. Padua L, Di Pasquale A, Pazzaglia C, Liotta GA, Librante A, Mondelli M. Systematic review of pregnancy-related carpal tunnel syndrome. Muscle Nerve 2010, 42 : 697-702.

16. Shiri R. Hypothyroidism and carpal tunnel syndrome : a meta-analysis. Muscle Nerve 2014, 50 : 879-83.

17. Eslamian F, Bahrami A, Aghamohammadzadeh N, Niafar M, Salekzamani Y, Behkamrad K.

Electrophysiologic changes in patients with untreated primary hypothyroidism. J Clin Neurophysiol 2011 Jun, $28: 323-8$.

18. Palma G. Carpal tunnel syndrome and hyperparathyroidism. Ann Neurol 1983, $14: 592$.

19. Kaku M, Berk JL. Neuropathy associated with systemic amyloidosis. Semin Neurol 2019, 39 : 578-588.

20. Sperry BW, Reyes BA, Ikram A, Donnelly JP, Phelan D, Jaber WA. Tenosynovial and cardiac amyloidosis in patients undergoing carpal tunnel release. J Am Coll Cardiol 2018, 72 : 2040-2050.

21. Donnelly JP, Hanna M, Sperry BW, Seitz WH Jr. Carpal tunnel syndrome : a potential early, red-flag sign of amyloidosis. J Hand Surg Am 2019, 44 : 868-876.

22. Maincent K, Héron B, Billette de Villemeur T, Mayer M. Early detection of median nerve compression by electroneurography can improve outcome in children with mucopolysaccharidoses. Orphanet J Rare Dis $2018,13: 209$.

23. Shiri R. Arthritis as a risk factor for carpal tunnel syndrome : a meta-analysis. Scand J Rheumatol 2016, $45: 339-46$.

24. Huang WH, Hu CC, Yen TH, Hsu CW, Weng CH. Blood lead level : an overlooked risk of carpal tunnel syndrome in hemodialysis patients. Ren Fail 2019, 41 : 786-793.

25. Fujita K, Kimori K, Nimura A, Okawa A, Ikuta Y. MRI analysis of carpal tunnel syndrome in hemodialysis patients versus non-hemodialysis patients : a multicenter case-control study. J Orthop Surg Res 2019, $14: 91$.

26. Thomsen NOB, Andersson GS, Björk J, Dahlin LB. Neurophysiological recovery 5 years after carpal tunnel release in patients with diabetes. Muscle Nerve 2017, 56 : E59-E64.

27. Panosyan FB, Kirk CA, Marking D, Reilly MM, Scherer SS, Shy ME, Herrmann DN. Carpal tunnel syndrome in inherited neuropathies: A retrospective survey. Muscle Nerve 2018, 57 :388-394.

28. Padua L, Padua R, Aprile I, Pasqualetti P, Tonali P, Italian CTS Study Group. Carpal tunnel syndrome. Multiperspective follow-up of untreated carpal tunnel syndrome : a multicenter study. Neurology 2001, $56: 1459-66$.

29. Agence nationale d'accréditation et d'évaluation en santé. Stratégie des examens paracliniques et des indications thérapeutiques dans le syndrome du canal carpien. Paris : ANAES, 1997.

30. American Academy of Neurology. Practice parameter for carpal tunnel syndrome (summary statement). Report of the Quality Standards Subcommittee of the American Academy of Neurology. Neurology 1993, 43 : 2406-9.

31. Calandruccio JH, Thompson NB. Carpal tunnel syndrome : making evidence-based treatment decisions. Orthop Clin North Am 2018, 49 : 223-229.

32. Piazzini DB, Aprile I, Ferrara PE, Bertolini C, Tonali P, Maggi L et al. A systematic review of conservative treatment of carpal tunnel syndrome. Clin Rehabil 2007, 21 : 299-314. 
33. O'Connor D, Marshall SC, Massy-Westropp N, Pitt V. Non-surgical treatment (other than steroid injection) for carpal tunnel syndrome. Cochrane Database of Systematic Reviews 2003, Issue 1. Mise à jour 2012.

34. Roquelaure Y, Ha C, Fouquet N, Descatha A, Leclerc A, Goldberg M et al. Attributable risk of carpal tunnel syndrome in the general population: implications for intervention programs in the workplace. Scand J Work Environ Health 2009, 35 : 342-348.

35. Chesterton LS, Blagojevic-Bucknall M, Burton C, Dziedzic KS, Davenport G, Jowett SM et al. The clinical and cost-effectiveness of corticosteroid injection versus night splints for carpal tunnel syndrome (INSTINCTS trial) : an open-label, parallel group, randomised controlled trial. Lancet 2018, 392 : 1423 1433.

36. Marshall S, Tardif G, Ashworth N. Local corticosteroid injection for carpal tunnel syndrome. Cochrane Database Syst Rev 2007, 2 : CD001554.

37. Jarvik JG, Comstock BA, Kliot M, Turner JA, Chan L, Heagerty PJ et al. Surgery versus non-surgical therapy for carpal tunnel syndrome : a randomised parallel-group trial. Lancet 2009, 374 : 1074-1081

38. Klokkari D, Mamais I. Effectiveness of surgical versus conservative treatment for carpal tunnel syndrome : a systematic review, meta-analysis and qualitative analysis. Hong Kong Physiother J 2018, 38 : 91-114.

39. Huisstede BM, Fridén J, Coert JH, Hoogvliet P, European HANDGUIDE Group. Carpal tunnel syndrome : hand surgeons, hand therapists, and physical medicine and rehabilitation physicians agree on a multidisciplinary treatment guideline-results from the European HANDGUIDE Study. Arch Phys Med Rehabil 2014, 95 : 2253-2263.

40. Haute Autorité de santé. Chirurgie du syndrome du canal carpien : approche multidimensionnelle pour une décision pertinente. Rapport d'évaluation. St Denis la Plaine : HAS, 2012.

41. Graham B, Peljovich AE, Afra R, Cho MS, Gray R, Stephenson J et al. The American Academy of Orthopaedic Surgeons evidence-based clinical practice guideline on : management of carpal tunnel syndrome. J Bone Joint Surg Am 2016, 98 : 1750-1754.

42. Atroshi I, Hofer M, Larsson GU, Ornstein E, Johnsson R, Ranstam J. Open compared with 2-portal endoscopic carpal tunnel release : a 5-year follow-up of a randomized controlled trial. J Hand Surg (Am) 2009, 34 : 266-272.

43. Agence nationale d'accréditation et d'évaluation en santé. Chirurgie du syndrome du canal carpien idiopathique : étude comparative des techniques à ciel ouvert et des techniques endoscopiques. Paris : ANAES, 2000.

44. Newington L, Brooks C, Warwick D, Adams J, Walker-Bone K. Return to work after carpal tunnel release surgery : a qualitative interview study. BMC Musculoskelet Disord 2019, $20: 242$.

45. Newington L, Francis K, Ntani G, Warwick D, Adams J, Walker-Bone K. Return to work recommendations after carpal tunnel release : a survey of UK hand surgeons and hand therapists. J Hand Surg Eur Vol 2018, 43 : 875-878. 